\title{
Synchronized Deformation Monitoring in Laser Cleaning: an Application for Cultural Heritage Conservation
}

\author{
Vivi Tornari*, Eirini Bernikola, Kostas Hatzigiannakis, Kristalia Melessanaki, Paraskevi Pouli \\ Institute of Electronic Structure and Laser, Foundation for Research and Technology-Hellas (IESL-FORTH), 71110 Heraklion, Crete, \\ Greece \\ *Corresponding Author: vivitor@iesl.forth.gr
}

Copyright (C) 2013 Horizon Research Publishing All rights reserved.

\begin{abstract}
The photo-induced effects generated upon laser cleaning result to the removal of unwanted material, still transient deformation and damage on the surface and the structure of the materials can be also occurred. It is herein presented a laser-based workstation to monitor deformation and to evaluate any damage potentially induced by laser cleaning processes in a non-destructive and on-line methodology. An all-optics laser geometry, which allows the alignment of the ablation beam and the monitoring system, is enabled. On-line surface monitoring of photomechanical effects is performed through the acquisition of surface condition and its relevant displacement associated to the synchronously performed laser cleaning procedure. Surface condition is traced by interference generated patterns expressed in spatial coordinates and their alterations throughout the cleaning process. Transient deformation, damage, successful or successful intervention is deduced upon comparison of the last to the initial-reference position of the surface points. The all-optics laser-workstation aims to respond to the increasing demand of controlled interventions satisfying safety regulations and preventive measures to be taken on time. As a proof of application principle infrared (IR) laser-cleaning in the removal of varnish from wooden objects monitored with the synchronized coherent interferometry system, is presented.
\end{abstract}

Keywords Holographic Interferometry, Digital Holographic Speckle Pattern Interferometry, Laser Cleaning, Cultural Heritage, Structural Diagnostics

\section{Introduction}

For a safe and successful intervention by means of laser-cleaning the main prerequisite is the correct choice of laser operation parameters. These parameters, being laser wavelength, laser fluence, pulse duration, repetition rate etc., are chosen in practice through preliminary tests on the basis of post-evaluation of the chemical and structural modifications and the morphology of the irradiated sample surface. Despite any thorough post-evaluation protocol, the results are not always clarifying up the optimum operation conditions, given that preliminary tests are only indicative of the actual situation. Since the aim of the application is to guarantee the optimum possible output to the sensitive surfaces of Cultural Heritage $(\mathrm{CH})$, the importance of developing monitoring procedures and devices becomes imperative.

In particular, in the conservation of $\mathrm{CH}$ the removal of unwanted surface layers (due to ageing, weathering or past treatments) is a demanding and delicate interventive action. Surface cleaning improves the aesthetic of the object, reveals hidden details, restores and eventually preserves it for the generations to come. It is one of the most essential and regularly performed restoration practices. Among the modern cleaning methods lasers hold a pioneer role as they enable high control and exceptional selectivity through the careful and thorough optimization of the ablation parameters [1]. In this respect the conservation community, following the state-of-the-art progress in laser studies during the past fifteen years, increasingly implements laser cleaning among their practices urging to bring standardized monitoring methods.

In this context the monitoring techniques implemented to safeguard the object against arbitrary and excessive actions should enable on-line observation and real-time feedback of the performed intervention. The possibility to provide immediate evaluation to adjust on-line the operation parameters requires synchronous operation of interventive and monitoring actions. Here interventive and monitoring processes are performed with optical non contact remote access methods hence the workstation is characterized as all-optics laser workstation.

\subsection{State of the Art in On-Line Monitoring}

The laser cleaning involves material removal with subsequent chemical and mechanical effects to the underlying or the surrounding areas. For the former, laser spectroscopic techniques, mostly breakdown spectroscopy (LIBS) or laser fluorescence (LIF), are used to identify the 
chemical composition of the involved materials and allow in-depth stratigraphy of multilayered surfaces $[1,2]$. The fluorescent spectra and the laser ablation plume spectra follow on-line the cleaning process, with the condition that the removed over-layers show different fluorescence characteristics or chemical composition than the substrate. Specifically, in CH conservation, LIBS can guide the depth of cleaning since by providing in-depth profiling of the diverse layers, highly accurate stratigraphy and ablation-spot analysis is obtained [3-5]. Multi-spectral imaging techniques enable in real time the visual observation of the surface during the cleaning process [6], and optical coherent tomography (OCT) techniques are successfully tested to follow the removal of thin varnish layers [7].

The photomechanical effects of the shock-waves propagation generated upon laser ablation may potentially provoke structural modifications to the surface, interfaces and to the bulk of the object. These potential effects cannot be detected by spectroscopy monitoring.

The expected photomechanical effects can primarily affect the structural integrity provoking bond-breaking and stress concentration among layers. The layer instability provokes three-dimensional changes in the object with most intense being the changes in axial direction. Consequently the on-line monitoring in structural terms of investigation requires tools sensitivity to dimensional changes. The requirement is satisfied if observation of changes lies on the central axis and the displacement sensitivity in axis perpendicular to the monitoring unit. By using a laser beam to probe the surface of the irradiated sample the surface displacement can be used to visualize changes both in the bulk and on the surface.

The laser interferometry techniques satisfy the above conditions and visualize the structural state without interacting with the sample. Different optical interferometric geometries provide the required sensitivity [8]. In general interferometric sensitivity is high and best suited for demanding spatial monitoring. In partial processes where only some part of the material layer is removed the induced rupture provokes the destabilization to the rest of surface layer and the underlying layers endangering the object's integrity [9]. The photothermal and photochemical processes are studied within the ablation spot or the ablated plume but in terms of photomechanical effects the ablation spot is shown as a static permanent damage. Thus the photomechanical phenomena are studied outside the ablation spot. As the mechanical wave propagates the structural and the mechanical properties of the surface are affected with static and dynamic changes occurring in long-term.

Therefore the photomechanical effects differ from the dominant laser ablation effects and require different investigation approach. These are studied simultaneously to the full extent of the surface of the irradiated sample and in temporal monitoring intervals. Photomechanical effects due to generation of structural flaws in solid materials are developed both in short and long-term and are monitored periodically opposed to photochemical and photothermal that are studied instantly [10 - 14].

\subsection{Previous Interferometry Studies on Photomechanical Effects of Laser Ablation}

Examination of photomechanical alterations was performed on polymer technical samples by means of optical holographic interferometry (HI) and Digital Holographic Speckle Pattern Interferometry (DHSPI), which allowed real-time visualization of the treated surfaces with resolving powers ranging from 100-3000 lines/ $\mathrm{mm}$ depended upon optics-geometry setting [9, 15-18]. The involved surface monitoring techniques offer the advantage of interferometric recording providing a particularly sensitive tool in deformation measurement in terms of high information content of the order of $0.2-0.3 \mu \mathrm{m}$ with the added property of holographically modulated interferometric field offering the $\mathrm{z}$ sensitivity vector of the displacement during the interferometry process [10]. Interferometry monitoring studies confirmed the temporal unfolding of effects with propagation of existed flaws and the dependency of flaw generation on material properties, while revealing the cohesion properties among layers and the age aspect of the material as a key-factor in the evolution of structural defects $[9,15,16]$. These techniques are chosen to visualize in real-time the surface reaction to the explosive removal of material, to control on-line the performance of operation parameters, to provide a warning in case of extremities, and to assess potential propagation of mechanical modifications, either in short-term when appear on-line, or in long-term during post-treatment examination.

In this context a controlled platform to perform the investigation and to standardize the laser intervention based on surface spatial constants is developed. The intervention is examined on-line for potential structural impact by monitoring the whole extent of the treated surface in full-field including the ablation spot.

\section{System and Methodology}

The laser cleaning \& monitoring integrated workstation is based on a prototype design of an all-in-one rigid-skeleton multifunctional base [30]. The all-in-one rigid-skeleton base is designed to accommodate all laser modules for modular spectroscopic analysis, multispectral imaging and interferometric monitoring in one rigid base with interchanging separate monitoring levels and x. y. z positioning. In this compact design instead of moving the plane of the object holder it is introduced a movable metal frame holding modular levels movable in $\mathrm{x}, \mathrm{y}, \mathrm{z}$ translation. Figure 1 shows the laboratory workstation scheme combining two levels of optical units; one to manipulate and direct the laser cleaning beam and the other one to monitor interferometrically the ablation process, a third optical unit not shown in the figure is foreseen for imaging and spectroscopy monitoring whenever required. In this paper 
the structural monitoring is emphasized and presented as it imposes most critical boundary conditions due to requirement for full-field long-term recording and spatiotemporal image constrains. The positioning system for the sample ablation is designed as stable feature. The synchronized laser cleaning beam scans on the sample's surface either manually or through a PC driven option, depending on the samples' material and morphology characteristics, while the structural evaluation of the cleaning process is automatically performed through custom-made acquisition algorithms, in order to allow visualization of the deformation of the irradiated surface at any instance of recording.

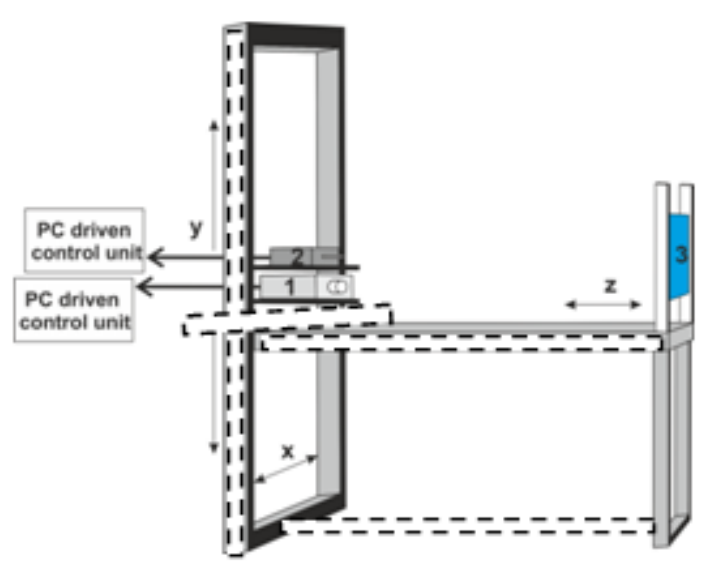

Figure 1. Schematic drawing of the synchronised $x, y, z$ workstation: 1) the DHSPI sensor, 2) laser-cleaning scanning device, 3) the monitored object under investigation

The rigid-skeleton base carrying the instrumentation warrants that extraneous low-frequency external vibrations would influence simultaneously the optical units, minimizing thus the risk of procedure failure due to accidental movement of one of the modules. Equally, this one-rigid-skeleton base plays important role in the monitoring resolution, quality and efficacy, since the evaluation errors due to induced noise are limited and high resolution requirements can be achieved. The irradiated surface (3) is placed perpendicularly to the laser ablation beam (2) facing the interferometry monitoring (1) beam. The structural monitoring beam divergence varies since it depends on the target and investigation requirement. The ablation beam is within the field of view of the structural monitoring beam. The difference in the beam divergences allow flexibility in positioning of the two utilities whereas in the case of LIBS spectroscopy standard automated articulated arm is inserted in position.

The cleaning unit may be comprised either by a laser nozzle controlled manually by the user or by a PC driven scanning device based on mirrors moved by galvanometric motors, which drive the laser ablation beam. The second case of a PC driven automated scanning laser cleaning procedure refers to $2 \mathrm{D}$ flat treated surfaces meaning macroscopically a low value of height-differences in the encrustation as well as to the object shape. The 2D surface obeys consistency rules upon selection of irradiation parameters of the surface and thus cleaning using an automated scanning protocol, is possible and safe. In case of advanced height differences and 3D shapes manual cleaning is performed. During cleaning operation the monitoring head is steadily mounted on the XY translator to move relatively to the object whereas the $\mathrm{z}$ position to control the field-of-view is a maximalisation of the design; since in practice beam divergence is directly controlled by the optics of the interferometry system. Operation is externally controlled by remote control unit and specially developed software, both custom-made for the application [6]. The laser scanning unit is also directly connected to the laser cleaning system.

\subsection{Interference Monitoring Principle}

The interference monitoring is based on coherent holographic interferometry geometry further equipped with lens and digital imaging device. Holography and holographic interferometry are pure lensless wavefield physical interference processes [8]. The addition of a lens-depended imaging system and a digital capturing device (CCD) at the recording plane hybrid the system properties resulting to a digital image of holographically modulated interference among speckle fields. Hence the recording properties are described by the term Digital Holographic Speckle Pattern Interferometry (DHSPI). DHSPI differs from Holographic Interferometry in the plane of recording dominating the spatial frequency properties of the interference. Substantial difference in physical properties from the Electronic Speckle Pattern Interferometry (ESPI) is due to the optical geometry which dominates the properties of interference phenomenon [8, 14]. The optical geometry in DHSPI utilizes the interference laws which rule holographic superposition by driving a reference beam $\left(\mathrm{U}_{\mathrm{R}} \mathrm{x}, \mathrm{y}\right)$ with complex amplitude:

$$
U_{R}(x, y)=\alpha_{R} \cdot \exp \left(i 2 \pi f_{y}\right)
$$

to interfere with two or more object beams $\left(\mathrm{U}_{0} \mathrm{x}, \mathrm{y}\right)$ with complex amplitude each of :

$$
U_{o}(x, y)=\alpha_{o}(x, y) \cdot \exp \left[-i \phi_{o}(x, y)\right]
$$

with $\alpha$ amplitude and the spatial frequency of reference beam being:

$$
f_{y}=\sin \theta_{R} / \lambda
$$

and the intensity distribution being:

$$
\begin{aligned}
& I(x, y)=\left|U_{o}+\alpha_{R} \exp \left(i 2 \pi f_{y}\right)\right|^{2} \\
& =\left|U_{o}\right|^{2}+\alpha_{R}^{2}+\alpha_{R} U_{o} \exp \left(-i 2 \pi f_{y} y\right) \\
& +\alpha_{R} U_{o}^{*} \exp \left(i 2 \pi f_{y} y\right)
\end{aligned}
$$


The equations describe the formation of interference fringes and the irradiation of the field. Holographic Interferometry is the science to create and utilize high spatial frequency interference phenomena. The speckle intensity fields' distributions as a function of time according to amplitude-division interferometry are utilized after the holographic interference has taken place by a Fourier transform lens as digital imaging system and the inverse numerical analysis of the captured speckle interference fields [31]. The number and interval of temporal separation of amplitude-splitted records is conditioned by the dynamics of the surface.

The surface under ablation is monitored by generating interference from its reference position and the following positions after induced material ablation. In order to follow alterations of the surface in consequential changes a carrier frequency is introduced by phase shifting the interference at each step of acquisition by $\pi / 2$ phase difference:

$$
\phi\left(\mathrm{m}, \mathrm{n}, \mathrm{t}_{0}\right)=\arctan \left(\frac{2\left(I_{2}-I_{4}\right)}{2 I_{3}-I_{5}-I_{1}}\right)
$$

The interferograms are produced having modulated phase $\varphi\left(\mathrm{m}, \mathrm{n}, \mathrm{t}_{0}\right)$ with $\mathrm{m}, \mathrm{n}$ be discrete values related to pixel position.

\subsection{Recording Technique and Experimental Procedure}

The surface under investigation is imaged onto a CCD camera by a lens producing a "speckled" image of the object with speckle being inherent to coherent illumination beam. Since speckle has finite size depending on the wavelength, the image distance and the aperture the spatial frequency of the interference should match the technical specification of the pixels of CCD. In the DHSPI portable interferometry system spatial frequency in terms of imaging resolution is on medium level with pixel size is $6.45 \mu \mathrm{m}$ and optimum speckle size is calculated as $b_{\mathrm{sp}}=12.9 \mu \mathrm{m}$.

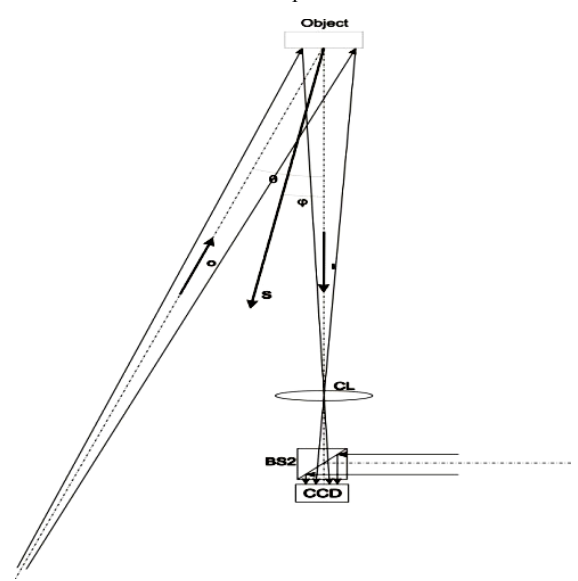

Figure 2. Schematic representation of the recording sensitivity vector employed in DHSPI system

In DHSPI each record is a light intensity distribution expressed in spatial coordinates allowing tracing surface point position within fractions of micrometers [32]. Thus with each surface condition that is recorded the intensity distribution is partially altered the dynamical changes are monitored and archived over time. The recording geometry as shown in figure 2 is a vector product of illumination and recording beams laying perpendicular to surface enabling sensitivity to out-of-plane deformations, with reference out-of-plane deformation at $\mathrm{z}$ position being $\mathrm{z}=0$.

In this figure $\mathrm{O}$ is the $O B$ object beam unitary vector and I is the backscattered OB beam unitary vector. The I is on the $\mathrm{z}$ axis which is the Object-CCD axis and the displacement sensitivity lies on the vector $\mathrm{S}$ of the $\mathrm{O}-\mathrm{I}$ beam. The sensitivity vector is:

$$
\mathrm{S}=2 \pi / \lambda(\mathrm{O}-\mathrm{I})
$$

With $\theta \cong 5^{\circ}$ the angle between vectors is $175^{\circ}$. In this case $\cos \theta$ is approximately $\cos \theta \approx-1$ and consequently the O-I vector size is calculated by

$$
|\mathrm{O}-\mathrm{I}|=1-\cos \theta=2
$$

leading to

$$
\mathrm{S}=4 \pi / \lambda
$$

Additionally with $\theta \approx 0$ where a is the angle between $\mathrm{S}$ and $\mathrm{Z}$ axis and then the phase difference is calculate

$$
\Delta \varphi=\mathbf{d} \mathbf{S}
$$

If object to recording plane geometry accounts for $\mathrm{S}$ on the $\mathrm{Z}$ axis only out of plane displacement is taken into account, that is $d=d_{z}$ then $\mathrm{S}$

$$
\Delta \varphi=\mathrm{Sd}_{\mathrm{z}}
$$

Hence the phase difference is given by

$$
\Delta \varphi=(4 \pi / \lambda) d_{z}
$$

For $\Delta \varphi=2 \pi$, that is one fringe pair difference $\mathrm{d}_{\mathrm{z}}=\lambda / 2$ which is the actual DHSPI resolution to out of plane displacements.

In DHSPI in order to calculate $\Delta \varphi$ and consequently the relative displacement, phase shifting has been applied and a 5-step algorithm has been used based on equation 5 .

The resulting two interferograms are further subtracted to give the fringe pattern of the phase change. The image produced from subtraction is then normalised using an 8-bit scale, in order to use the full dynamic range of the image format, and subsequently filtered to increase the fringe visibility (contrast enhancement)[20-22].

The automated scanning cleaning unit (2 in Fig. 1) is based on mirrors driven through galvanometric motors. A custom-made software controls the relative movement of the laser beam in respect to the object following the determined cleaning parameters (number of pulses, percentage of overlapping between adjacent areas etc). For the cleaning tests a Q-switched Nd:YAG laser system (Spectron SL805) was employed. The system emits pulses of $10 \mathrm{~ns}$ at $1064 \mathrm{~nm}$. Irradiation experiments were performed on a XY scanning mode using fluence values in the range of $0.8-2 \mathrm{~J} / \mathrm{cm}^{2}$. The beam was weakly focused to a $1.7-2.2 \mathrm{~cm}^{2}$ elliptical surface, while the applied number of pulses varied from 1 up to 3 and the overlapping percentage varied from 30 to $50 \%$ in both 
directions due to the surface smooth texture that allows steady set of cleaning parameters.

The procedure followed in order to implement the synchronous operation in all-in-one workstation, suited at this phase to carry the on-line spatial monitoring of laser cleaning procedure, is described in table I.

$$
\begin{array}{l|c}
\multicolumn{1}{c}{\text { Table 1. Experimental Procedure }} \\
\text { i } & \begin{array}{c}
\text { A sequence of reference raw data of } \\
\text { interferograms is captured prior to laser } \\
\text { irradiation. }
\end{array} \\
\text { ii } & \begin{array}{c}
\text { Irradiation tests are performed in order to test a } \\
\text { variety of parameters (energy fluence values, } \\
\text { number of pulses, overlapping etc.) } \\
\text { iii }
\end{array} \\
\text { iv } & \begin{array}{c}
\text { Sets of interferograms are recorded after the } \\
\text { ablation tests. }
\end{array} \\
\text { v } & \begin{array}{c}
\text { assessed } \\
\text { the preliminary results were analyzed and } \\
\text { ai }
\end{array} \\
\text { vii } & \begin{array}{c}
\text { assefined } \\
\text { the optimum ablation parameters are defined } \\
\text { the optimum spatial acquision parameters are } \\
\text { on-line monitoring with simultaneous operation } \\
\text { of the laser cleaning system and the DHSPI } \\
\text { acquisition module is ready to be performed }
\end{array} \\
\text { Time duration of each surface record to next }<4 \text { sec }
\end{array}
$$

\subsection{Laser Cleaning of Shellac Films from Wood}

The laser assisted removal of polymeric protective coatings of natural (varnishes) or synthetic (i.e. consolidation materials) origin from $\mathrm{CH}$ surfaces is a well studied subject in the field [1, 23-28]. The layer-by-layer removal of well-defined layers of polymer without damaging the underlying sensitive surface (i.e. paintings) is usually achieved through ultraviolet laser ablation mechanisms. The removal of varnishes from wood is an interesting application given the stability and resistance of the wooden substrate which allows for employment of more efficient lasers emitting in the IR. Previous studies [29] have established the optimum laser parameters for the removal of such protective coatings from wood. Through comparative studies in a wide range of laser systems it was concluded that a successful and effective laser assisted removal of shellac from wood could
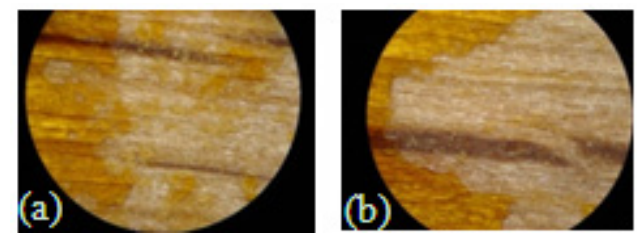

(a)

be achieved using the IR radiation emitted from a Q-Switched Nd:YAG laser system at $1064 \mathrm{~nm}$ at fluence values in the range of 1.2 to $1.8 \mathrm{~J} / \mathrm{cm}^{2}$. Above these values damage to the wooden substrate could potentially occur while bellow $1.2 \mathrm{~J} / \mathrm{cm}^{2}$ varnish removal could not be feasible. Of course the number of applied pulses must be regulated according to the thickness of the protective film on the basis of the calculated etching rates in this regime [29].

For the measurements described in this paper a set of two wooden flat mock-ups $(13 \times 13 \times 0.6 \mathrm{~cm})$ were used. The two samples were made from two types of wood (pine and oak) and were coated by brush with shellac varnish on their tangential surface. The coating thickness was on the range of $20 \mu \mathrm{m}$ to $35 \mu \mathrm{m}$.

In figure 3 the cleaning tests on both samples are shown, while figure 4 shows close details of the ablated areas of the sample at different cleaning levels (optimum, under- and over- cleaned). Optimum- cleaned areas refer to areas in which laser ablation parameters were successfully chosen so as to remove homogeneously the bulk of the varnish layer without any damaging effects to the underlying wood surface, as confirmed by close observations using an optical microscope (OM) (Figure 4b). On the other hand, under-cleaned areas (Figure 4a) refer to areas in which the laser ablation parameters (laser fluence, applied number of pulses and/or overlapping parameters) could not lead to a successful and homogeneous varnish removal. Finally irradiation at fluence values over the ablation threshold or at an excessive number of pulses causes damage to the wooden substrate (Figure 4c). The different classes of laser ablation threshold in cleaning appoint the detection sensitivity thresholds of the monitoring

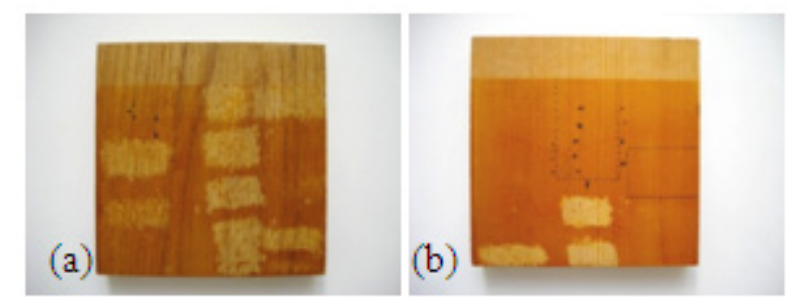

Figure 3. the technical samples employed in this study (a) Sample 1- oak with shellac and (b) Sample 2- pine with shellac.

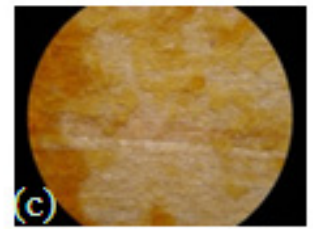

Figure 4. Macrophotographs of (a) undercleaned (sample 1-scan $5, \mathrm{~F}=1.1 \mathrm{~J} / \mathrm{cm}^{2}, 6$ pulses), (b) optimum cleaned (sample 1 - scan $8, \mathrm{~F}=1.6 \mathrm{~J} / \mathrm{cm}^{2}, 2 \mathrm{pulses}$ ) and (c) overcleaned-damaged (sample 2 -scan $9, \mathrm{~F}=1.85 \mathrm{~J} / \mathrm{cm}^{2}, 2$ pulses) areas 

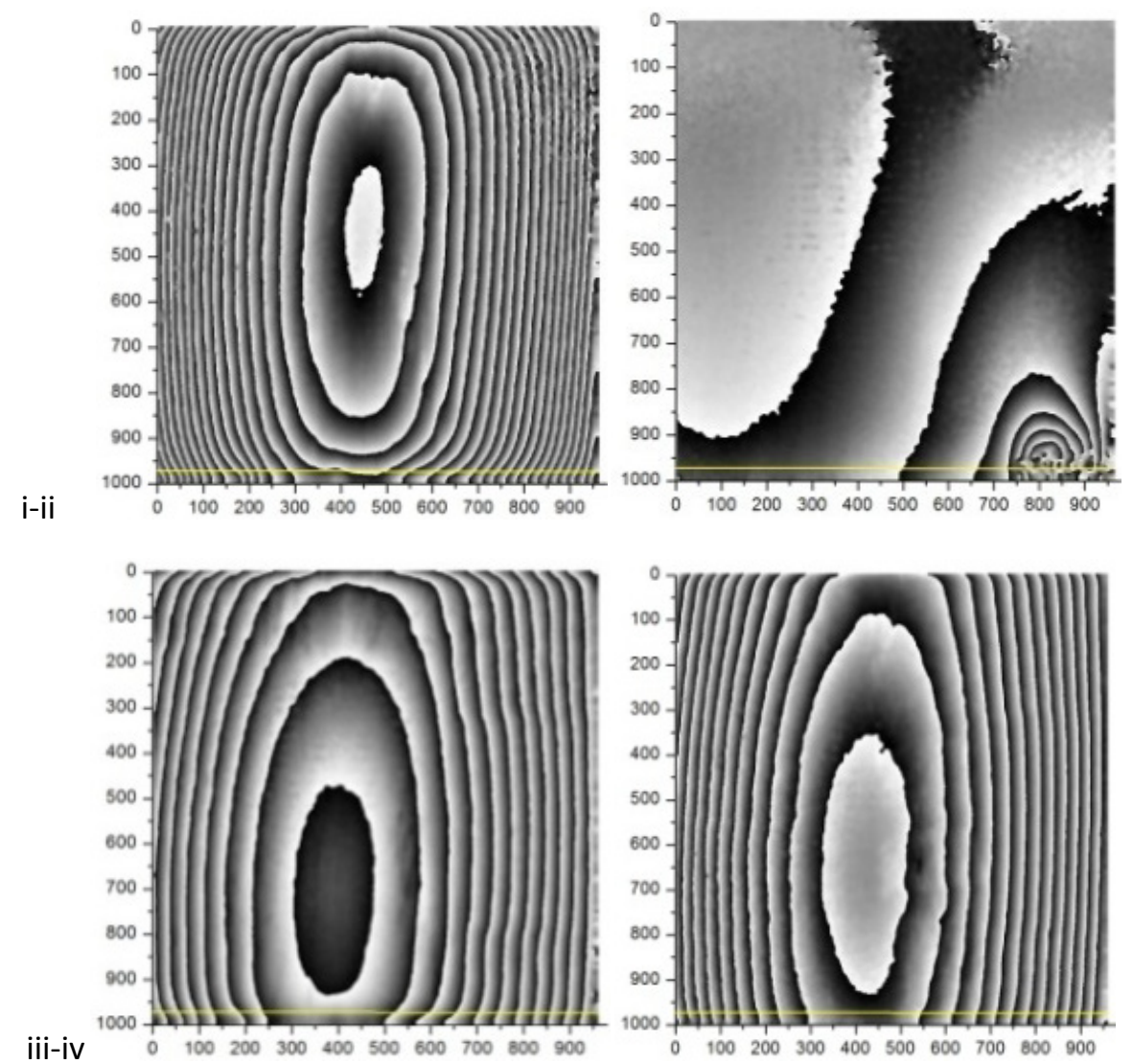

Figure 5. Interferograms captured at $80 \mathrm{sec}$ upon excitation (i) before laser cleaning, (ii) during laser cleaning, (iii) after laser cleaning and (iv) 1 month after laser cleaning

Workstation design allows monitoring for long-term photomechanical effects generated from materials flaws [30-34]. The samples have been monitored at (i) reference condition before laser intervention, (ii) during laser intervention, (iii) after laser cleaning and (iv) 30 days after the experiment for all three cases ((a) undercleaned, (b) optimum cleaned and (c) overcleaned). Case (a): under-cleaned corresponds to sample 1, scanned area 5 in which irradiation took place with 6 pulses of $\mathrm{F}=1.1 \mathrm{~J} / \mathrm{cm}^{2}$, Case (b): optimum cleaned corresponds to sample 1, scanned area 8 with 2 pulses of $\mathrm{F}=1.6 \mathrm{~J} / \mathrm{cm}^{2}$, and Case (c): over-cleaned corresponds to sample 2 , scanned area 9 with 2 pulses of $\mathrm{F}=1.85 \mathrm{~J} / \mathrm{cm}^{2}$.

\section{Results and Discussion}

A sequence of interferograms has been captured for each cleaned area at all four experimental conditions (i-iv) and the intensity profiles for case (a), case (b) and case (c) are presented. Intensity profiles along the same pixel coordinates visualize dark to bright fringe distribution. They correspond to a display of a gray-value profile of each interferogram in exact pixel position.

\footnotetext{
3.1. CASE (a): sample 1 , scanned area 5 with $F=1.1 \mathrm{~J} / \mathrm{cm}^{2}$, 6 pulses
}

In all four experimental conditions, a sequence of interferograms has been recorded with a step of four seconds between each interferogram. In experimental conditions (i), (iii) and (iv) IR heating has been used as the excitation method in order to achieve the recording of the deformed state of the object whereas in experimental condition (ii) the laser irradiation from the laser cleaning device acts as the excitation method. Figure 5 (i-iv) shows interferograms captured at the same time interval $(80 \mathrm{sec})$ upon excitation while in figure 1 the intensity profiles corresponding to measurements of pixel intensity along a selected raw which corresponds to the middle (in $\mathrm{X}$ axis) of the area under investigation (row 972, marked with a yellow line) are presented. The resolution of the pictures of interferograms is $990 \times 1000$ pixels. The specific coordinates define the intensity profile across the centre of the cleaned area.

In graph of figure 6 are visualised the intensity profiles, before cleaning (i) the marked area which undergone the laser cleaning process (ii) whereas in intensity profiles corresponding to measurements obtained immediately and one month after the laser cleaning (iii, iv), the area is unaffected by the cleaning procedure. The laser ablation pulses affected only transiently the surface and no damage has been occurred due to the laser cleaning process. In this case the cleaning parameters, fluence and number of pulses, are considered to be not efficient for the cleaning of the area. 


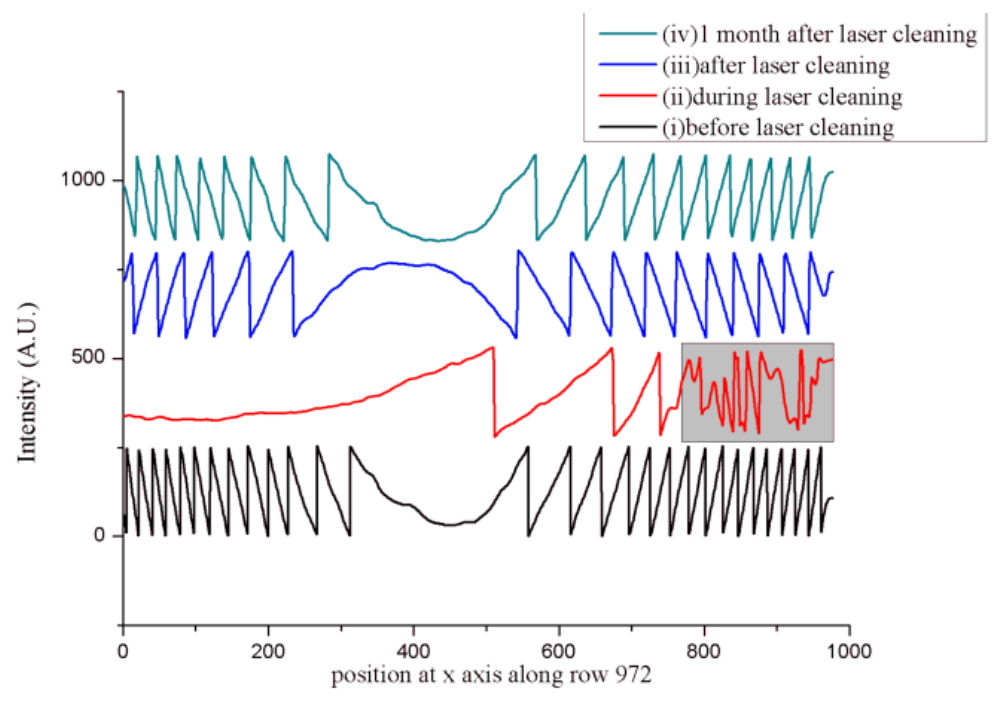

Figure 6. Intensity profiles along row 972 for (i) before laser cleaning, (ii) during laser cleaning, (iii) after laser cleaning and (iv) 1 month after laser cleaning

\subsection{CASE (b): sample 1, scanned area 8 with $F=1.6 \mathrm{~J} / \mathrm{cm}^{2}, 2$ pulses}

The monitoring process on sample 1 following same procedure as previously was re-examined with new cleaning parameters. Figure 7 (i-iv) shows interferograms captured at the same time interval $(80 \mathrm{sec})$ upon excitation while in figure 8 the intensity profiles corresponding to measurements of pixel intensity along a selected row (row 628, marked with a yellow line) are presented.
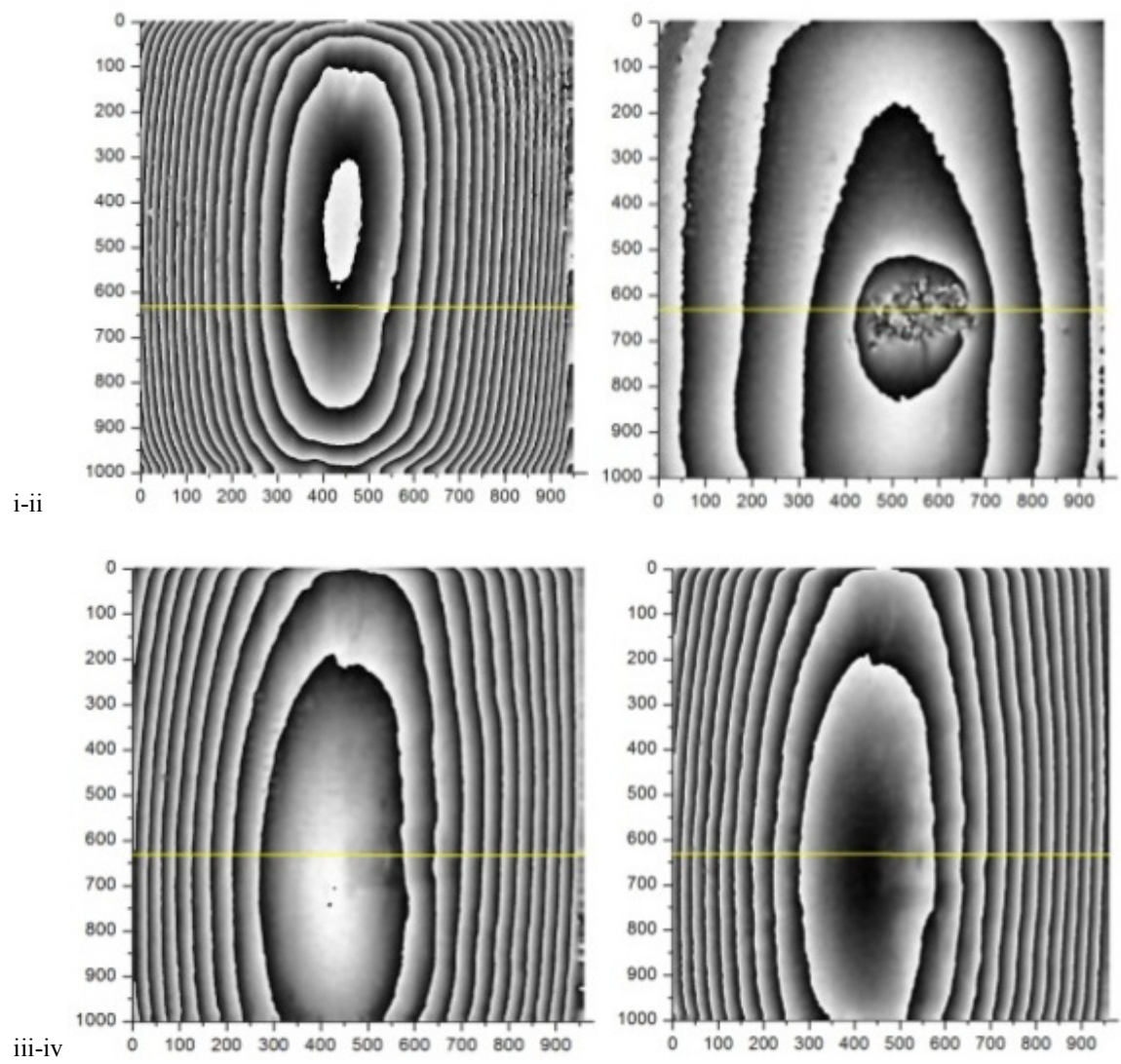

Figure 7. Interferograms captured at 90sec upon excitation (i) before laser cleaning, (ii) during laser cleaning, (iii) after laser cleaning and (iv) one month after laser cleaning 
As observed in graph of figure 8 the area where the laser cleaning has been performed is again marked in the intensity profile corresponding to the laser cleaning procedure (ii) whereas in intensity profiles corresponding to measurements obtained after the laser cleaning procedure (iii, iv) the area is not being affected. In this case further examination of the sample leads to the conclusion that the laser cleaning process was successful whereas the intensity profiles confirm the fact that no damage has been occurred during the cleaning process employing optimum cleaning conditions in the investigated area.

\subsection{CASE (c): sample 2 , scanned area 9 with $F=1.85 J /$ $\mathrm{cm}^{2}, 2$ pulses}

Exactly the same procedure as before has been followed with excessive laser cleaning parameters as in the "over-cleaned" case. Figure 9 (i-iv) shows interferograms captured at the same time interval $(80 \mathrm{sec})$ upon excitation while in figure 10 the graphs of the intensity profiles corresponding to measurements of pixel intensity along a selected row (row 756, marked with a yellow line) are presented.

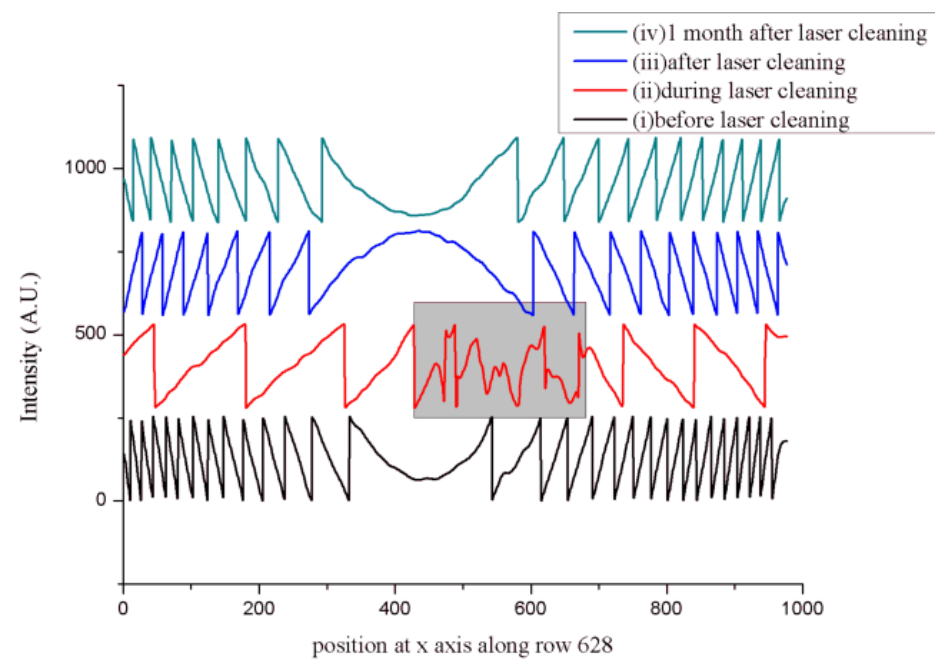

Figure 8. Intensity profiles along row 628 for (i) before laser cleaning, (ii) during laser cleaning, (iii) after laser cleaning and (iv) 1 month after laser cleaning

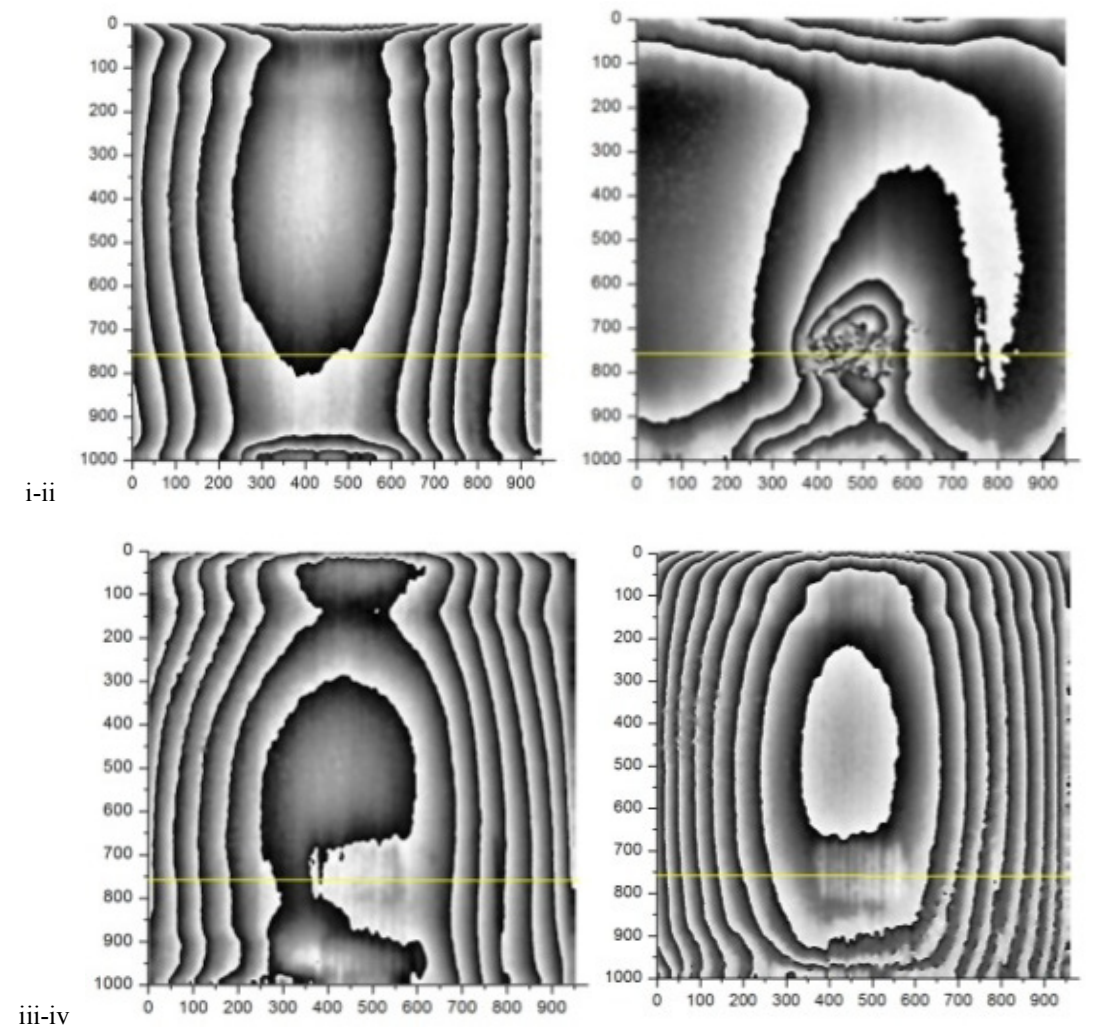

Figure 9. Interferograms captured at 90sec upon excitation (i) before laser cleaning, (ii) during laser cleaning, (iii) after laser cleaning and (iv) 1 month after laser cleaning 


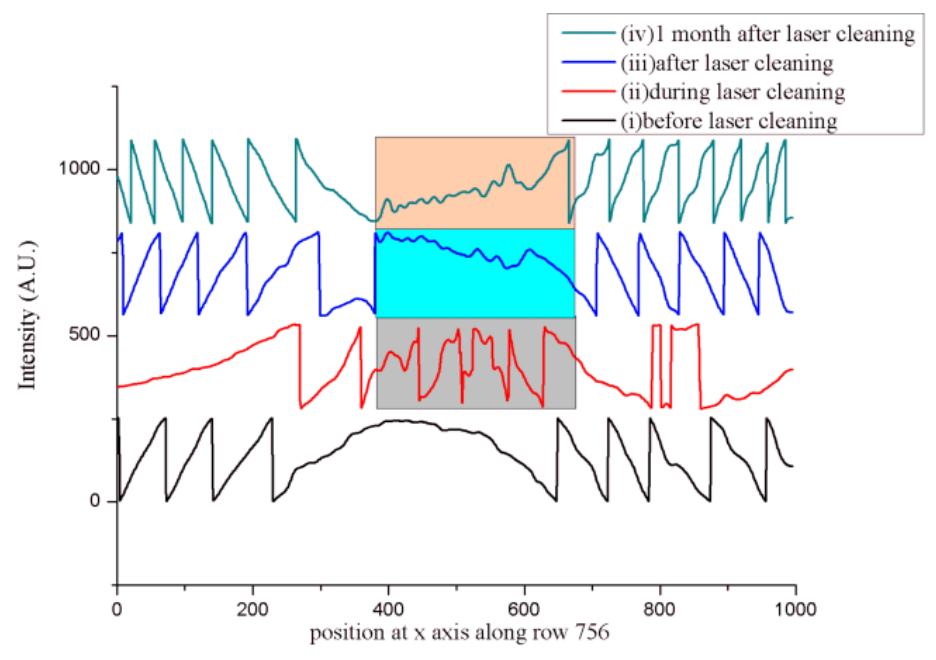

Figure 10. Intensity profiles along row 756 for (i) before laser cleaning, (ii) during laser cleaning, (iii) after laser cleaning and (iv) 1 month after laser cleaning

The affected area by the laser cleaning process has been marked in intensity profiles (ii), (iii) and (iv) where it has been observed that the homogeneity of the intensity profiles (iii) and (iv) is disturbed in the same area where the laser cleaning has been performed. The damage caused upon excessive laser cleaning parameters can be also visualized and confirmed via the 3-D representation of the captured interferogram after 1 month that corresponds also to the deformation of the sample (Figure 11).

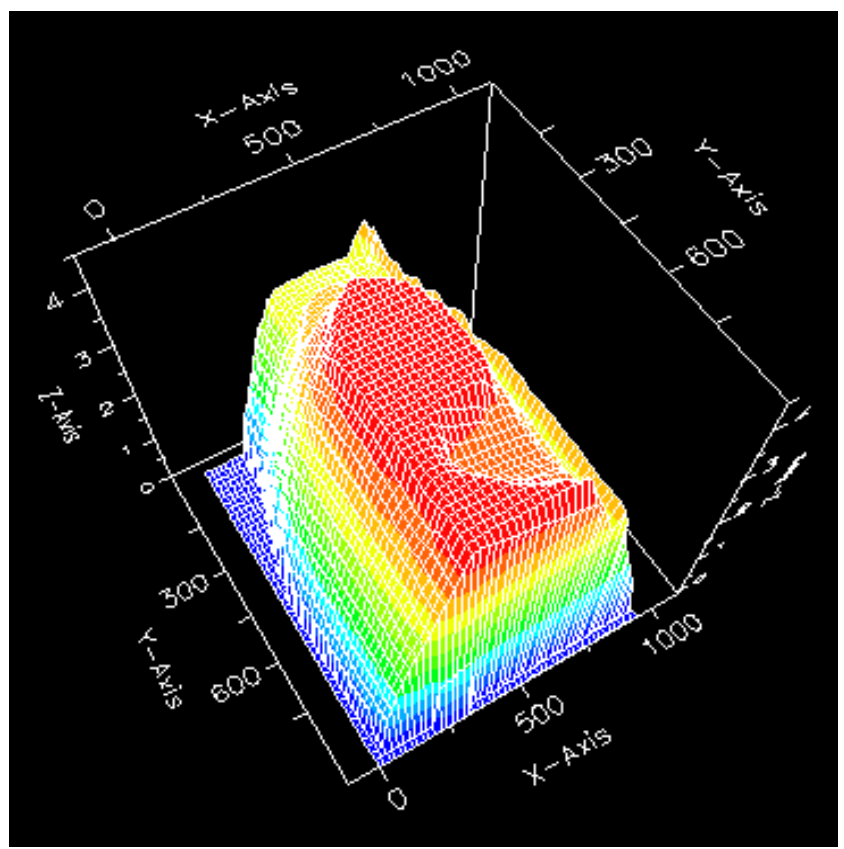

Figure 11. 3-D representation of interferogram captured 1 month after laser cleaning intervention

The resulted interferograms obtained during the three tests of laser cleaning processes that correspond to cases (a)-(c) (namely as under-cleaned, optimum cleaned and over-cleaned) have been examined and the relative displacement of the object in each case has been calculated and presented in figure 12 .

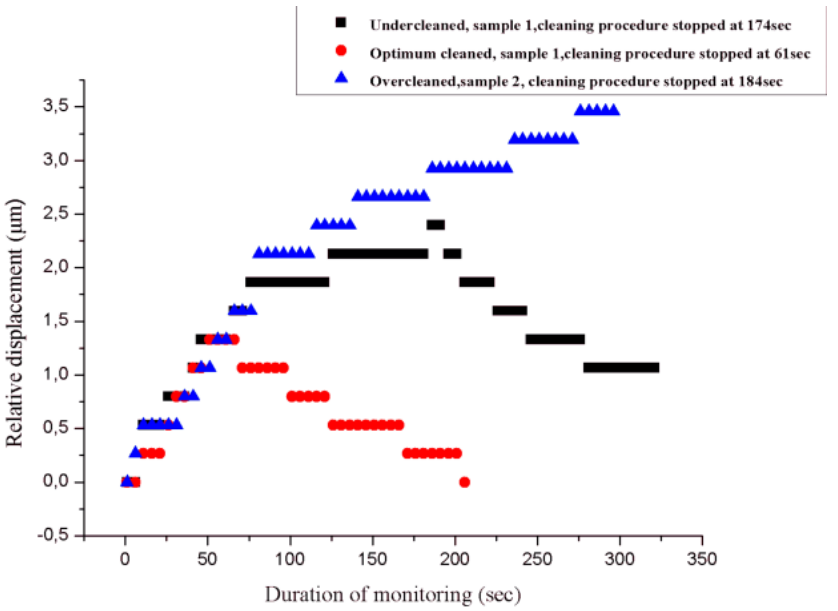

Figure 12. Relative displacement curves vs duration of monitoring during laser cleaning processes for (i) Under-cleaned, (ii) Optimum cleaned and (iii) Over-cleaned

As observed, the relative displacement that corresponds to undercleaned (cleaning procedure stopped at $174 \mathrm{sec}$ ) and optimum cleaned (cleaning procedure stopped at $61 \mathrm{sec}$ ) demonstrates a very similar behavior. The pick of the curve is being located at the point where the laser cleaning process stops and follows a relaxation decrease. On the contrary, in the relative displacement curve that corresponds to over-cleaned (cleaning procedure stopped at $184 \mathrm{sec}$ ) the point of the curve where the laser cleaning process stops is not being followed by a relaxation curve but the displacement continues to increase. The recorded responses of the surface displacement could be thus considered as a confirmation of the existence of the damage caused by the laser cleaning process.

\section{Conclusions}

A synchronized deformation monitoring of laser cleaning 
interventions by an all-optics monitoring procedure carried on an integrated rigid skeleton base is presented and discussed in this paper. The experimental setup, as well as, a methodology for the parallel application of herein presented structural monitoring with Digital holographic Speckle Pattern Interferometry system and the laser cleaning process is presented and discussed.

The potential of using interference principle with DHSPI system as a surface monitoring tool to detect on-line induced structural modifications due to laser cleaning is confirmed. Through systematic tests on technical samples of shellac coated wooden samples it was shown that exaggerated cleaning conditions, resulting into damaged surfaces, could be clearly distinguished over the optimized or under-cleaned ones and thus a protocol of laser based structural monitoring of laser cleaning can be developed. Further studies on laser ablation spot are underway in order to quantify this methodology and thus to calibrate the synchronized procedure.

Furthermore, other diverse geometries involving the relative position of the ablation and the monitoring of laser beams are discussed in order to accommodate different cleaning case problems (i.e. in opposite directions to detect deformation in canvases, or in certain relative angles in case of irregular surface relieves, or multiple angles in case of complicated shapes eg. statues, furniture).

It has been shown a workstation which allows simultaneous conservation actions for monitoring laser cleaning process and probable induced effects either in transient or damaging level. The application is thus expected to allow the minimization of undesired effects or potential damages and to optimize the safeguarding procedures. In cleaning practices it is expected to allow freedom of choice to the conservator and expand the applicability of the lasers in conservation procedures by facilitating and ensuring the introduction of laser-cleaning applications in the artwork and antiquities conservation.

\section{REFERENCES}

[1] Fotakis C, Anglos D, Zafiropulos V, Georgiou S, Tornari V. 2007, Lasers in the Preservation of cultural heritagePrinciples \& Applications, New York: Taylor \& Francis.

[2] Anglos D. Laser-induced breakdown spectroscopy in art \& archaeology. Appl. Spectrosc 2001; 55:186A.

[3] Gobernado-Mitre I, A. Prieto C, Zafiropulos V, Spetsidou Y, Fotakis C. On-line monitoring of laser cleaning of limestone by laser-induced breakdown spectroscopy and laser-induced fluorescence. Appl. Spectrosc. 1997; 51:1125-1129.

[4] Melessanaki K, Stringari C, Fotakis C, Anglos D. Laser Cleaning and Spectroscopy: A Synergistic Approach in the Conservation of a Modern Painting. Laser Chemistry 2006, Article ID 42709.

[5] Fortes F.J, Cabalín L.M, Laserna J.J. The potential of laser-induced breakdown spectrometry for real time monitoring the laser cleaning of archaeometallurgical objects. SpectrochimicaActa Part B: Atomic Spectroscopy 2008; 63: 1191.

[6] Papadakis V, Loukaiti A, Pouli P. A spectral imaging methodology for determining on-line the optimum cleaning level of stonework. Journal of Cultural Heritage 2010; 11: 325-328.

[7] Targowski P, Góra M, Wojtkowski M. Optical Coherence Tomography for Artwork Diagnostics. Laser Chemistry 2006; Article ID 35373, 11 doi:10.1155/2006/35373

[8] Vest C. M. Holographic Interferometry, Wiley, N.Y., 1979.

[9] Bonarou A, Antonucci L, Tornari V, Georgiou S, Fotakis C. Holographic interferometry for the structural diagnostics of UV laser ablation of polymer substrates. Applied Physics A 2001; 73: 647-651.

[10] Tornari V, Zafiropulos V, Bonarou A, Vainos N.A, Fotakis C. Modern technology in artwork conservation: a laser-based approach for process control and evaluation. J. Opt. and Lasers in Engineering 2000; 34:309-326.

[11] Tornari V, Bonarou A, Zafiropulos V, Fotakis C, Smyrnakis $\mathrm{N}$, Stassinopoulos S. Structural evaluation of restoration processes with holographic diagnostic inspection. Journal of Cultural Heritage 2003; 4: S347-S354. Tornari V, Bonarou A, Zafiropulos V, Fotakis C, Doulgeridis M. Holographic applications in evaluation of defect and cleaning procedures. J. Cult. Heritage 2000; 1: S325-S329.

[12] Tornari V, Orphanos Y, Dabu R, Blanaru C, Stratan A, Ursu D. Non-destructive speckle interferometry diagnosis method for art conservation. Proceedings SPIE Vol. 6606,Advanced Laser Technologies 2006, Dan C. Dumitras; Maria Dinescu; Vitally I. Konov, Editors, 66060W, 25 April 2007.

[13] Tornari V. Laser Interference-Based Techniques and Applications in Structural Inspection of Works of Art. Analytical and Bioanalytical Chemistry2007;387: 761-80.

[14] Dabu R, Blanaru C, Pacala O, Stratan A, Fenic C, Neagu L, Rusen L, Ursu D, Tornari V, Orphanos Y. Single-frequency green nanosecond Nd:YAG microlaser-oscillator power-amplifier system. Intnl. Conference Micro- to Nano-Photonics ROMOPTO 2006, Aug. 28-31, 2006, Sibiu, ROMANIA, SPIE volume 6785, paper number 08.

[15] Tornari V, Bonarou A, Antonucci L. Sequential holographic interferometric recording: a key to monitor dynamic displacements in long-term effects. 4th International workshop on automatic processing of fringe patterns, Bremen 17-19 September 2001, Elsevier FRINGE 2001, pp 680-685

[16] Athanassiou A, Andreou E, Bonarou A, Tornari V, Anglos D, Georgiou S, Fotakis C. Examination of chemical and structural modifications in the UV ablation of polymers. Applied Surface Science 2002; 197-198: 757-763.

[17] Tsiranidou E, Tornari V, Orphanos Y, Kalpouzos C, Stefanaggi M. Time-dependent defect reveal assessed by combination of laser sensing tools. In the Proceedings of the 6th International Conference on Lasers in the Conservation of Artworks (LACONA VI): 611-620.

[18] Schnars U, Jueptner W. Digital Holography, Springer.

[19] Hariharan P. Basics of Interferometry 2nd ed - WW 2007: 
209-210.

[20] Kreis K. Handbook of Optical Interferometry - (Wiley-VCH, 2005) WW: 254-255.

[21] Chih-Cheng Kao, Gym-Bin Yeh, Shu-Sheng Lee, Chih-Kung Lee, Ching-Sang Yang, and Kuang-Chong Wu. Phase-shifting algorithms for electronic speckle pattern interferometry. Appl. Optics 2002; 41-1: 46-54

[22] Pouli P, Nevin A, Andreotti A, Colombini P, Fotakis C. Laser assisted removal of synthetic conservation materials from paintings using UV radiation of ns and fs pulse duration; morphological studies on model samples. Applied Surface Science 2009; 255: 4955-4960.

[23] Pouli P, Paun I.-A, Bounos G, Georgiou S, Fotakis C. The potential of UV femtosecond laser ablation for varnish removal in the restoration of painted works of art. Applied Surface Science 2008; 254- 6875-6879.

[24] Melessanaki K, Stringari C, Fotakis C, Anglos D. Laser Cleaning and Spectroscopy: A Synergistic Approach in the Conservation of a Modern Painting. Laser Chemistry 2006, Article ID 42709, 2006. doi:10.1155/2006/42709.

[25] Pouli P, Melessanaki K, Giakoumaki A, Argyropoulos V, Anglos D. Measuring the thickness of protective coatings on historic metal objects using nanosecond and femtosecond LIBS depth profiling. Spectrochimica Acta Part B 2005; 60-1163-1171.

[26] Lassithiotaki M, Athanassiou A, Anglos D, Georgiou S,
Fotakis C. Photochemical Effects in the UV Laser Ablation of Polymers: Implications for Laser Restoration of Artworks. Appl. Phys. A 1999; 69 -363-67.

[27] Georgiou S, Zafiropulos V, Anglos D, Balas C, Tornari V, Fotakis C. Excimer laser restoration of painted artworks: Procedures, Mechanisms and Effects. Applied Surface Science 1998; 127-129- 738-45.

[28] Aligizaki E.M, Melessanaki, K. Pournou A. The use of lasers for the removal of shellac from wood. e-PS 2008; 5-36-40.

[29] V. Tornari, "Applications of Holographic Interferometry in Preventive Conservation and the Diagnosis of Works of Art", MPhil Thesis, V\&A/RCA \& British Libraries, (UK), 1996

[30] Osten W. and Juptner W., Digital Processing of Fringe Patterns, in Handbook of Optical Metrology, Rastogi P. K., Editor. 1997, Artech House Publishers: Boston and London.

[31] V. Tornari, "Spatial Coordinates in Interferometry Fringes: A Timeless Artwork Multipurpose Documentation", Journal of basic and applied physics, Aug. 2012, Vol. 1, Iss. 2, pp. 39-48

[32] V. Tornari, "Laser Interference-Based Techniques and Applications in Structural Inspection of Works of Art", Analytical and Bioanalytical Chemistry; 387, 761-80 (2007)

[33] Vivi Tornari, Eirini Bernikola, Elsa Tsiranidou, "Interference fringe-patterns association to defect-types in artwork conservation: an experiment and research validation review", Applied Physics A: Volume 106, Issue 2 (2012), Page $397-410$ 